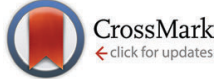

Cite this: J. Mater. Chem. C, 2015, 3, 3946

Received 12th February 2015, Accepted 1st March 2015

DOI: $10.1039 / \mathrm{c} 5 \mathrm{tc0} 0435 \mathrm{~g}$

www.rsc.org/MaterialsC

\section{Stability and electronic properties of silicene on $\mathrm{WSe}_{2}$}

\author{
Jiajie Zhu and Udo Schwingenschlögl*
}

\begin{abstract}
Many semiconducting substrates, such as $\mathrm{GaS}$ and $\mathrm{MgBr}_{2}$, have been explored for silicene. However, large lattice mismatches, complicated control of terminal layers and small band gaps are critical limiting factors. First-principles results on the stability and electronic properties of silicene on $\mathrm{WSe}_{2}$ show that the energy barriers for lateral translation between the two subsystems are very small due to weak van der Waals interactions. For the same reason, the Dirac physics of silicene is preserved. It turns out that the induced band gap is sufficient to withstand thermal fluctuations.
\end{abstract}

\section{Introduction}

Silicene, first proposed theoretically by Takeda and Shiraishi in $1994,{ }^{1}$ consists of a monolayer of $\mathrm{Si}$ atoms in a two-dimensional honeycomb structure, just like graphene. Even though freestanding silicene is subject to buckling $\left(\mathrm{sp}^{2}-\mathrm{sp}^{3}\right.$ hybridization), ${ }^{2,3}$ the Dirac cone at the corner of the Brillouin zone (linearly dispersing $\pi$ and $\pi^{*}$ bands) is maintained. ${ }^{4}$ In addition to its electronic similarity to graphene, ${ }^{5-7}$ silicene is also suitable to be integrated in current Si-based nanoelectronics. Furthermore, the stronger spin-orbit coupling as compared to graphene opens new avenues for applications. ${ }^{8}$

Although silicene has been successfully deposited on several metallic substrates, including $\mathrm{ZrB}_{2}(0001),{ }^{9} \operatorname{Ir}(111)^{10}$ and $\mathrm{Ag}(111),{ }^{11,12}$ the Dirac characteristics are destroyed because of hybridization of $\pi$ bands with the substrate. ${ }^{13-15}$ On the other hand, semiconducting substrates have been investigated in order to avoid such strong interaction. For example, GaS nanosheets have been predicted to yield a Dirac cone with a $170 \mathrm{meV}$ band gap, while the lattice mismatch of $7.5 \%$ casts doubts on the stability of this hybrid system. ${ }^{16}$ Kokott and coworkers have found for silicene on $\mathrm{H}(\mathrm{Cl})$-passivated $\mathrm{Si}(111)$ substrate (lattice mismatch less than $1 \%$ ) linearly dispersing $\pi$ bands with a band gap of 3(56) meV. ${ }^{17,18}$ Similar results have been reported for $\mathrm{H}$-passivated Si- and C-terminated $\operatorname{SiC}(0001) \cdot{ }^{19,20}$ Although Dirac physics can be achieved on $\mathrm{MgX}_{2}(0001)(\mathrm{X}=\mathrm{Cl}, \mathrm{Br}$ and I) substrates without dangling surface bonds, which simplifies the synthesis, band gaps below $16 \mathrm{meV}$ strongly limit the applicability. ${ }^{21} \mathrm{~A}$ band gap of $52 \mathrm{meV}$ is induced by F-terminated $\mathrm{CaF}_{2}(111)$, but control of the preparation process is very problematic. ${ }^{18}$

PSE Division, King Abdullah University of Science and Technology, Thuwal 23955-6900, Kingdom of Saudi Arabia. E-mail: udo.schwingenschlogl@kaust.edu.sa
A band gap of some $100 \mathrm{meV}$ is typically desirable to overcome thermal fluctuations at room temperature. Several approaches have been studied to open such a band gap in graphene, including external electric fields ${ }^{22,23}$ and multilayer stacking, ${ }^{24,25}$ which may also be useful for silicene. However, the experimentally accessible electric fields limit the band gap to about $30 \mathrm{meV} \cdot{ }^{26,27}$ Moreover, adjacent Si layers are predicted to form clusters instead of bilayer silicene, which completely destroys any Dirac states because of the strong interaction. ${ }^{21,28}$ While adsorbed metal atoms are predicted to open a band gap in freestanding silicene, they may aggregate instead of maintaining a uniform distribution. ${ }^{29,30} \mathrm{WSe}_{2}$ is a semiconductor with a hexagonal structure consisting of sandwich-like layers along the [001] direction without dangling bonds. ${ }^{31}$ It has minimal lattice mismatch with low-buckled silicene among the transition metal dichalcogenides, consequently overcoming the practical obstacles caused by related substrates with much larger lattice mismatch. ${ }^{32}$ Since the van der Waals interaction is expected to be stronger than for $\mathrm{MgBr}_{2}$ (more electrons are contributing to the dipole), the band gap of silicene on $\mathrm{WSe}_{2}$ is expected to be enhanced. Therefore, $\mathrm{WSe}_{2}$ appears to fulfill all criteria of a suitable substrate for silicene. We will investigate in this work the stability and electronic properties of this hybrid system and will demonstrate very encouraging results.

\section{Computational method}

Electronic structure calculations are performed in the framework of density functional theory and the projector augmented wave method..$^{33}$ The generalized gradient approximation of Perdew, Burke and Ernzerhof is employed for the exchange-correlation potential $^{34}$ and the long-range van der Waals interaction is taken into account by the DFT-D2 method. ${ }^{35}$ The cut-off energy 
(a)

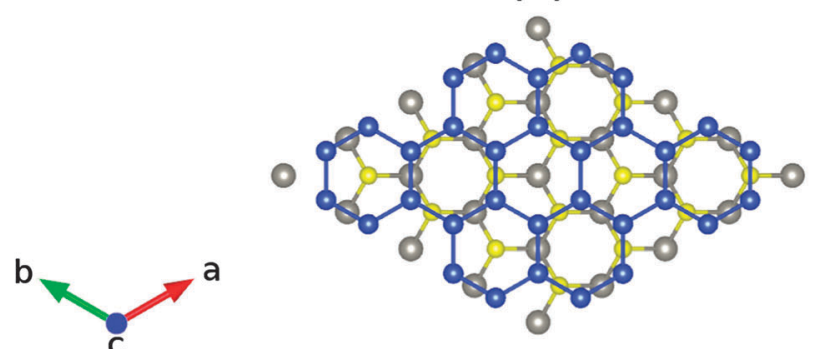

(c)

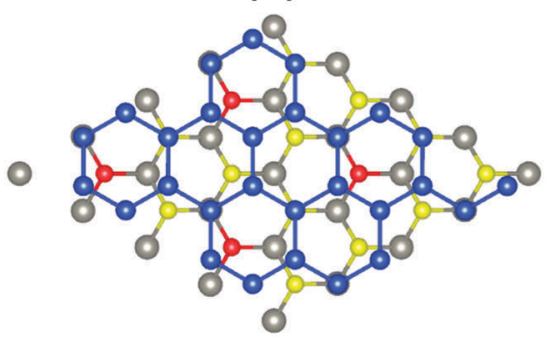

(b)

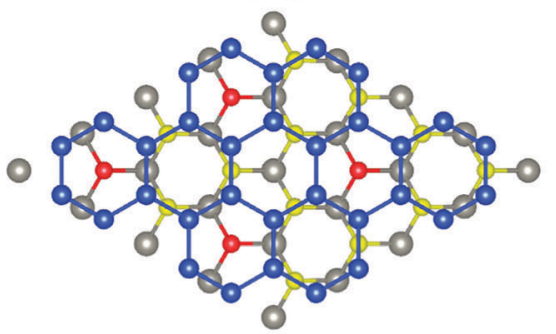

(d)

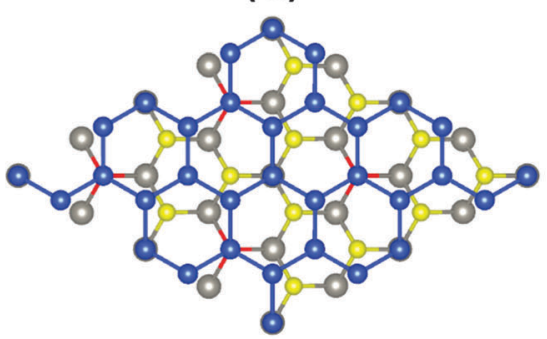

(e)

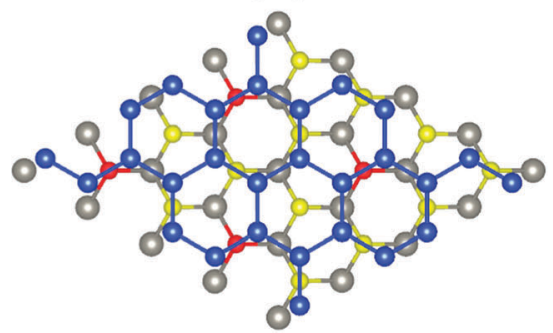

Fig. 1 Top view of low-buckled silicene on (a) undoped WSe $\mathrm{W}_{2}$ without lateral shift and (b-e) S/Te doped WSe $\mathrm{e}_{2}$ with lateral shifts of $0,1 / 6,1 / 3$ and $1 / 2$ along the [100] direction. The W, Se, Si and S/Te atoms are shown in gray, yellow, blue and red color.

for the plane wave basis is set to $400 \mathrm{eV}$ and an energy tolerance of $10^{-6} \mathrm{eV}$ is selected for the iterative solution of the KohnSham equations. Brillouin zone integrations are performed with $9 \times 9 \times 1 \mathrm{k}$-meshes in the structure optimizations and $18 \times 18 \times 1 k$-meshes in the electronic structure calculations. All structures are relaxed until the residual forces on the atoms have declined to less than $0.01 \mathrm{eV} \AA^{-1}$.

The in-plane lattice constant of $\mathrm{WSe}_{2}(001)$ is found to be $3.313 \AA$ and thus similar to the experimental and previous theoretical values of $3.282 \AA$ and $3.31 \AA$, respectively. ${ }^{36}$ The lattice mismatch between $2 \times 2 \mathrm{WSe}_{2}$ and $\sqrt{3} \times \sqrt{3} R\left(30^{\circ}\right)$ lowbuckled silicene is only $0.6 \%$. High-buckled silicene is also considered, since it has been prepared on $\mathrm{MoS}_{2} \cdot{ }^{37}$ Furthermore, monolayer $\mathrm{WSe}_{2}$ has been obtained by mechanical exfoliation..$^{38}$ We use a vacuum layer of $15 \AA$ thickness to avoid unphysical interaction between images due to the periodic boundary conditions. Dipole corrections result only in tiny energy variations of less than $0.1 \mathrm{meV}$ per atom. Low-buckled silicene on bilayer $\mathrm{WSe}_{2}$ is investigated for comparison with the monolayer case. In addition, $\mathrm{S}$ and $\mathrm{Te}$ atoms are substituted for

Table 1 Structural properties of low-buckled silicene on $\mathrm{WSe}_{2}$ without lateral shift: Si buckling height $\left(b_{\text {sil }}\right)$, distance between the silicene sheet and substrate $\left(d_{\text {sil-sub }}\right)$, lattice constant $(a)$, binding energy per Si atom $(E)$, formation energy of the dopant atom $(\Delta H)$ and bond distance between $\mathrm{W}$ and the dopant site $\left(d_{\mathrm{W} \text {-dop }}\right)$

\begin{tabular}{llll}
\hline & S doping & Pristine & Te doping \\
\hline$b_{\text {sil }}(\AA)$ & 0.51 & 0.51 & 0.49 \\
$d_{\text {sil-sub }}(\AA)$ & 3.19 & 3.20 & 3.03 \\
$a(\AA)$ & 3.829 & 3.842 & 3.861 \\
$E(\mathrm{meV})$ & 112 & 122 & 135 \\
$\Delta H(\mathrm{eV})$ & 0.01 & & 0.32 \\
$d_{\text {W-dop }}(\AA)$ & 2.42 & 2.54 & 2.73
\end{tabular}

Se (concentration $12.5 \%$ ) to modify the stability of the hybrid system as well as the band gap of silicene.
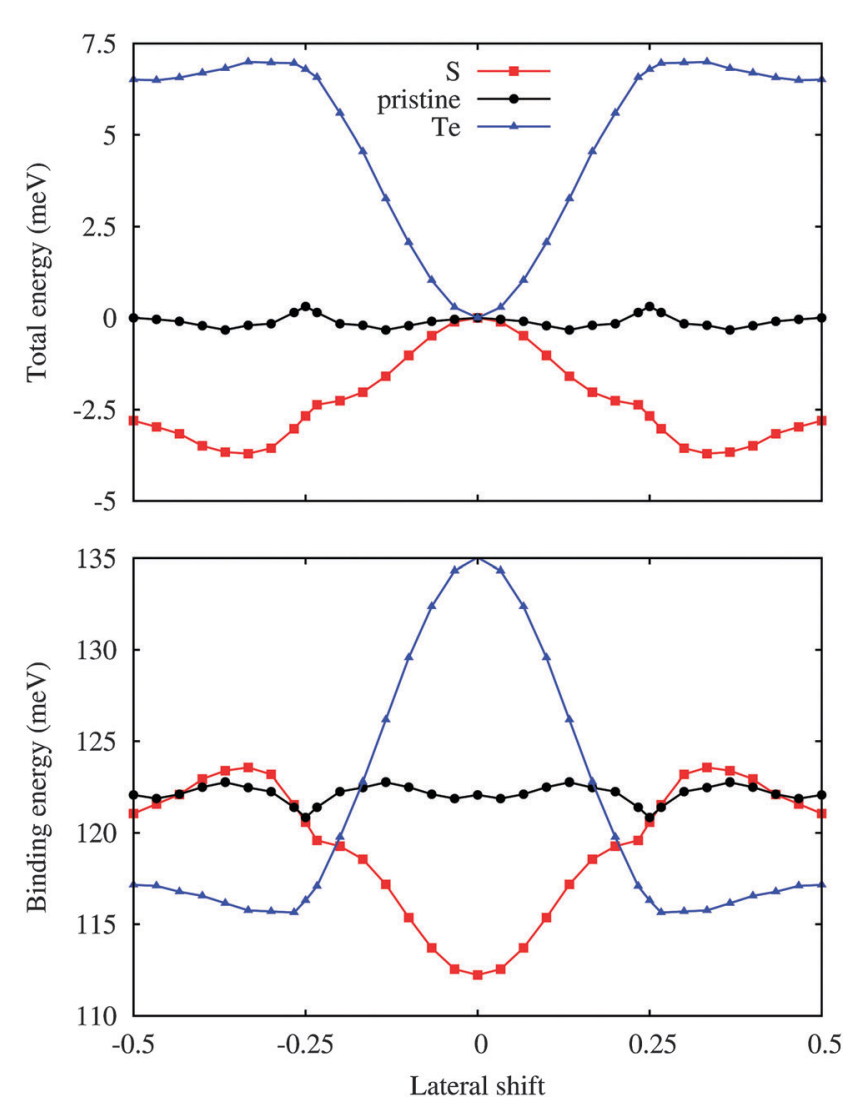

Fig. 2 Total energy per atom with respect to the unshifted structure and binding energy per Si atom for low-buckled silicene on $\mathrm{WSe}_{2}$ as a function of the lateral shift along the [100] direction. 


\section{Results and discussion}

Fig. 1(a) shows the structure of low-buckled silicene on monolayer $\mathrm{WSe}_{2}$, where the $\mathrm{W}$ and Se atoms are located on top of the hollow and bridge sites of the Si hexagons (which we will call in the following the unshifted structure). According to the structural parameters summarized in Table 1, the buckling height of the Si atoms ( $0.51 \AA)$ as well as the binding energy (122 meV) are larger than in the case of the $\mathrm{MgBr}_{2}$ substrate $(0.49 \AA$ and $63 \mathrm{meV})$ due to the stronger van der Waals interaction. ${ }^{21}$ Moveover, the binding energy is close to that of low-buckled silicene on $\mathrm{MoS}_{2}$ $(120 \mathrm{meV}) .{ }^{39} \mathrm{~A}$ bilayer $\mathrm{WSe}_{2}$ substrate yields little differences for the structural properties of silicene. The Si buckling height and in-plane lattice constant are virtually identical to the monolayer result, whereas the distance between the substrate and silicene grows by about $1 \%$ to $3.23 \AA$ A. Since the binding energy (121 meV) is also similar, we conclude that already the $\mathrm{WSe}_{2}$ monolayer accurately models realistic substrate thicknesses.

The variation of the total energy of the hybrid system as a function of the lateral shift along the [100] direction with respect to the unshifted structure is illustrated in Fig. 2. Note that the [100] and [010] directions are equivalent due to the three-fold rotational symmetry of the lattice. The unshifted structure in addition has mirror symmetry and the translational periodicity is half of the cell size due to the $2 \times 2 \mathrm{WSe}_{2}$ supercell. The maximal translation energy barrier is found to be less than $0.4 \mathrm{meV}$ per atom, which reflects a high uncertainty in the structure of the hybrid system at room temperature as well as below. The binding energy of the two components is also addressed in Fig. 2, showing a variation of less than $2 \mathrm{meV}$ per Si atom with distinct valleys at lateral shifts of \pm 0.25 along the [100] direction.

We next substitute $S$ or Te for Se to modify the interaction at the interface, see Fig. 1. Different configurations of the dopant atoms ranging from clusters to a homogeneous distribution are compared. The latter turns out to be energetically favorable due to small local distortions around the dopant atoms, which are located on top of the $\mathrm{Si}$ atoms for $1 / 6$ and $1 / 3$ lateral shifts, respectively, and occupy hollow and bridge sites for 0 and $1 / 2$ lateral shifts. The corresponding formation energies are 0.01 and $0.32 \mathrm{eV}$ (Table 1$)$. The $\mathrm{W}-\mathrm{S}(2.42 \AA)$ and $\mathrm{W}-\mathrm{Te}(2.73 \AA)$ bond lengths deviate from the $\mathrm{W}-$ Se bond length $(2.54 \AA)$ as expected from the ionic radii $(1.70,1.84$ and $2.07 \AA$ for $\mathrm{S}$, Se and Te, respectively). Smaller (3.829 $\AA$ ) and larger (3.861 $\AA$ ) in-plane lattice constants are predicted for $S$ and Te doping. In addition, the distance between silicene and the substrate remaines similar for S doping (3.19 $\mathrm{A}$ ) but is significantly reduced for Te doping (3.03 $\AA$ ), see Table 1 . For $S$ doping a $\pm 1 / 3$ lateral shift is energetically favorable, see Fig. 2, whereas the unshifted structure now has (a)

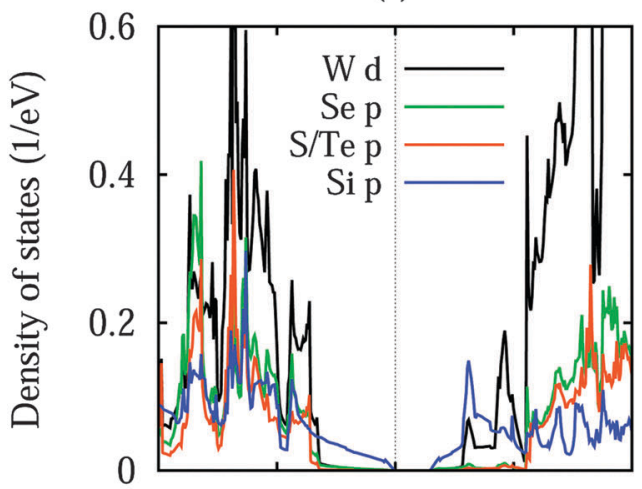

(d)

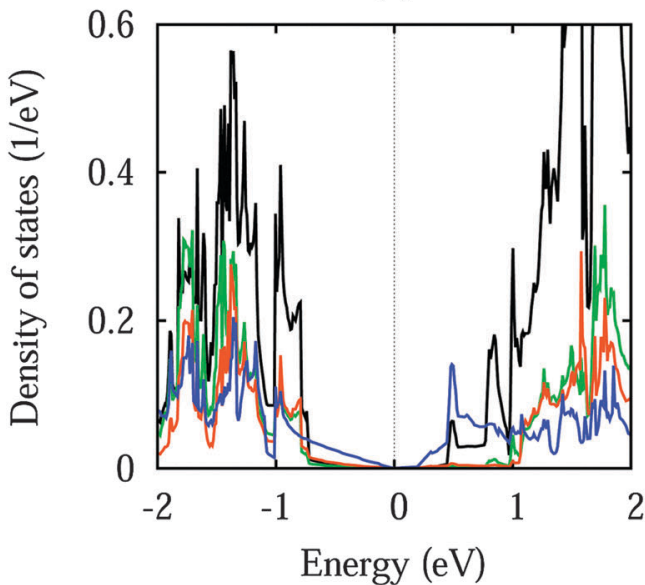

(b)

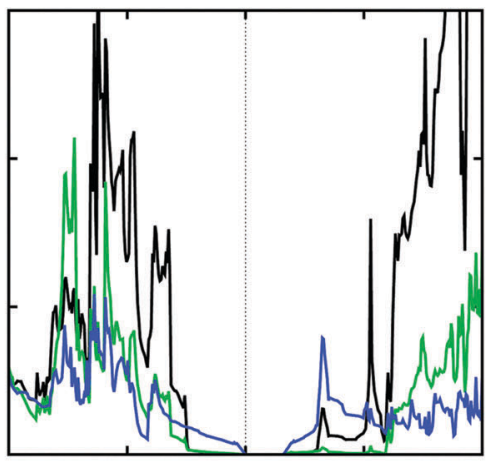

(e)

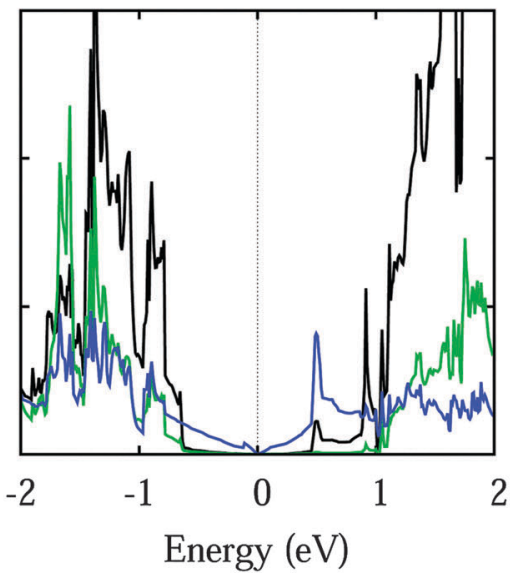

(c)

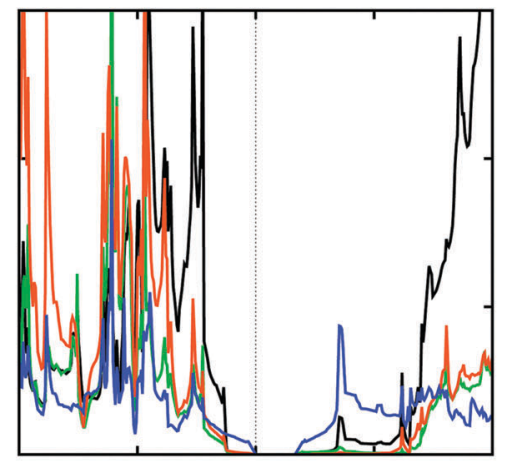

(f)

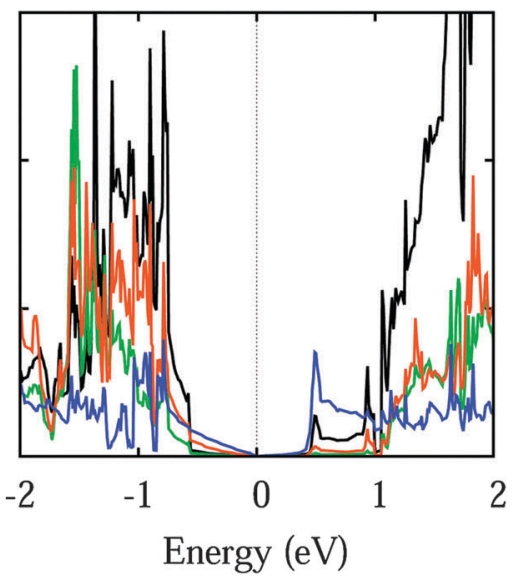

Fig. 3 Density of states of low-buckled silicene on (a, b) S doped, (c, d) pristine and (e, f) Te doped WSe 2 without lateral shift (left row) and with a $1 / 3$ lateral shift (right row). 
(a)

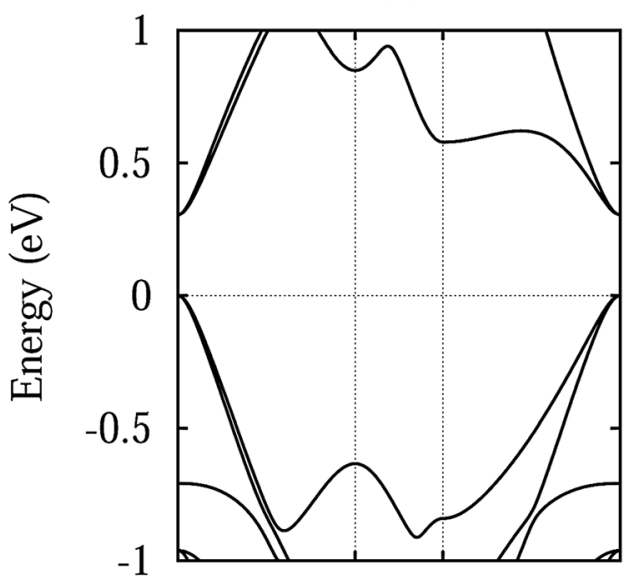

(d)

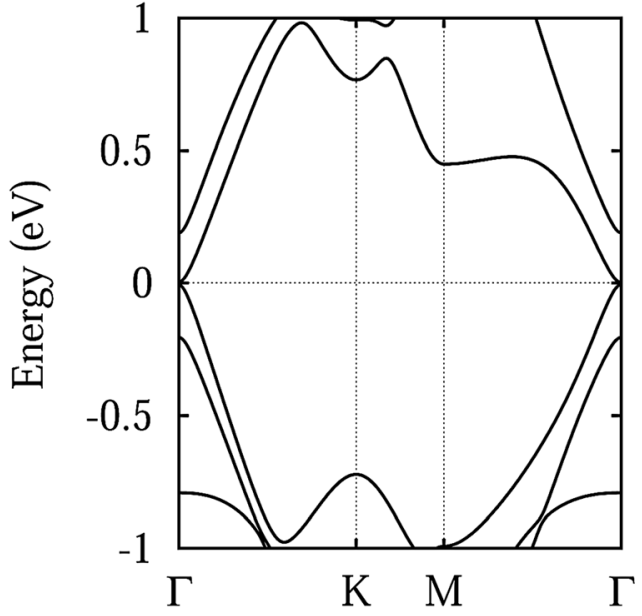

(b)

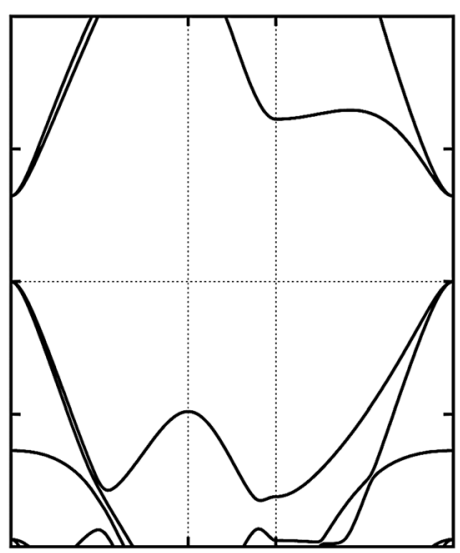

(e)

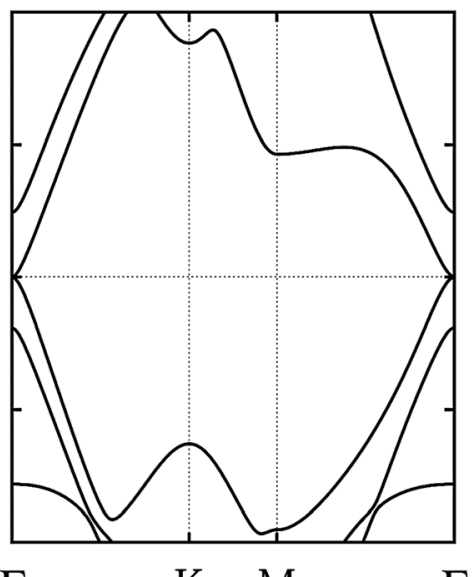

(c)

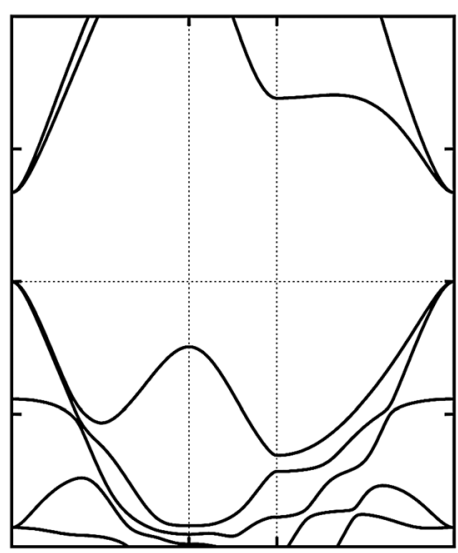

(f)

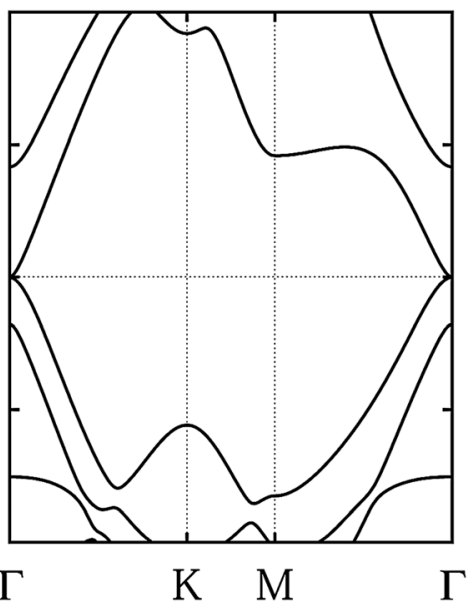

Fig. 4 Band structure of low-buckled silicene on (a, d) S doped, (b, e) pristine and (c, f) Te doped WSe $\mathrm{H}_{2}$ without lateral shift (upper row) and with a 1/3 lateral shift (lower row).

the highest total energy. The resistance against translation is enhanced substantially as compared to the pristine case, which is a consequence of stronger variations of the binding energy. Under Te doping the unshifted structure is most stable and the maximal translation energy barrier is enhanced to $7 \mathrm{meV}$, which corresponds to a binding energy difference of $19 \mathrm{meV}$ per Si atom (including $2 \mathrm{meV}$ of relaxation energy from the substrate).

Fig. 3 shows densities of states without shift and with a lateral shift of $1 / 3$ (most and least stable configurations for different dopings). The top of the valence band and the bottom of the conduction band are dominated by $\mathrm{Si} 3 \mathrm{p}$ states. Hybridization between the substrate and Si $3 p$ states extends closer to the valence band maximum for Te doping than for $\mathrm{S}$ doping due to the smaller distance to the silicene. Moreover, larger hybridizations between the $\mathrm{S} / \mathrm{Te} \mathrm{p}$ and $\mathrm{Si} 3 \mathrm{p}$ states are characteristic for the unshifted structures. The band structures in Fig. 4 show the Dirac cone, as expected, at the $\Gamma$ point. While alkali metal intercalation can also restore the Dirac cone of double-layered silicene, the present system is less difficult to handle experimentally. ${ }^{40}$ Without lateral shift we obtain band gaps of 0.30 , 0.32 and $0.34 \mathrm{eV}$ for $\mathrm{S}$ doped, pristine and Te doped $\mathrm{WSe}_{2}$.
The slight increase reflects the growing interaction at the interface, compare Table 1 . Spin-orbit coupling reduces the band gap of the pristine system without lateral shift by $2.8 \mathrm{meV}$ due to spin splitting (2.0 meV in the valence band and $1.5 \mathrm{meV}$ in the conduction band), which is negligible as compared to the original value. On the other hand, the band gap is close to zero for the shifted structures because of weaker interaction: in the unshifted case $\mathrm{S} / \mathrm{Se} / \mathrm{Te}$ occupies a hollow site and thus has six $\mathrm{Si}$ neighbours, whereas in the shifted case it occupies a top site with only a single interaction partner. Although Se vacancies are inevitable in monolayer $\mathrm{WSe}_{2}{ }^{41}$ they only slightly reduce the band gap, for example by $0.06 \mathrm{eV}$ for undoped $\mathrm{WSe}_{2}$ without lateral shift (one Se vacancy in a $4 \times 4$ supercell), and leave the principal features of the band structure unaffected.

The position of the Dirac point (middle point between the $\pi$ and $\pi^{*}$ bands) with respect to the Fermi level and the corresponding band gap are illustrated in Fig. 5. Doping is found to have hardly any effect on both these quantities. On the other hand, the band gaps are reduced to almost zero for the 1/6 and $1 / 3$ lateral shifts, which are structurally similar with the $\mathrm{W}$ and Se or dopant atoms on top of the Si atoms. Since the translation 

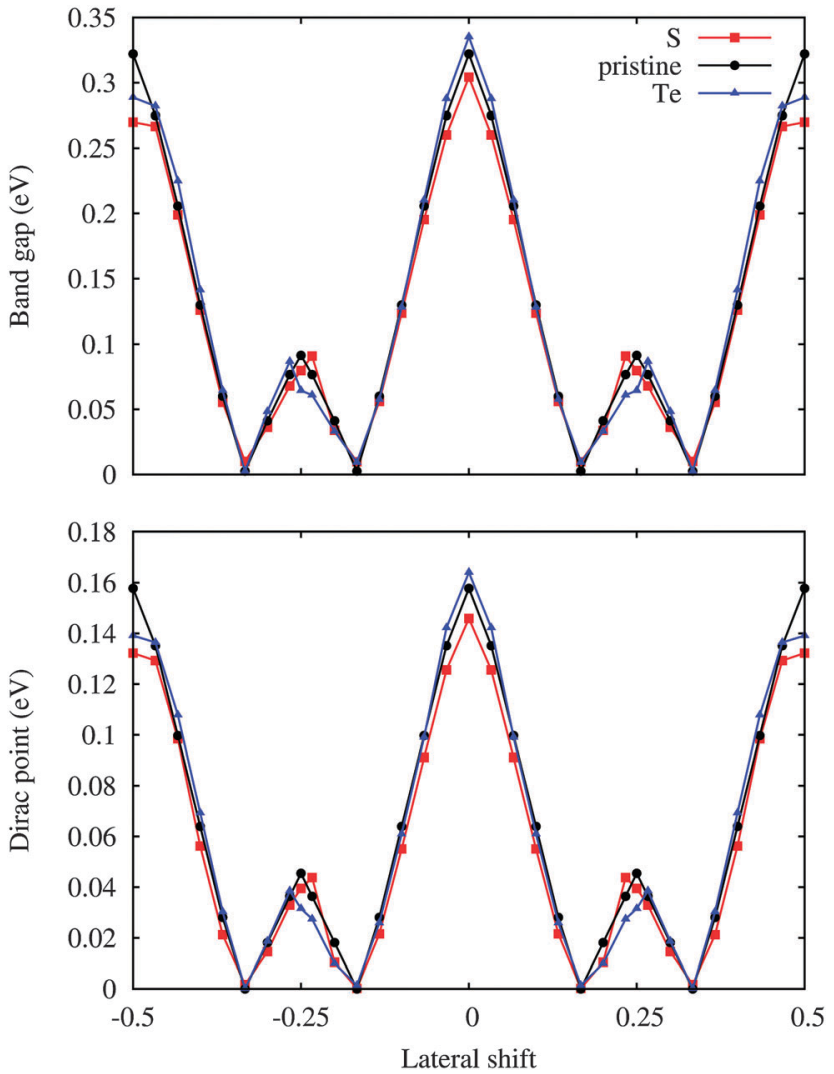

Fig. 5 Position of the Dirac point with respect to the Fermi level and band gap as a function of the lateral shift along the [100] direction for lowbuckled silicene. energy barrier is quite small for the pristine substrate, thermal fluctations even at low temperature can modify the electronic properties dramatically, ranging from metallic to semiconducting characters. On the contrary, stable metallic and semiconducting silicene is obtained by $\mathrm{S}$ and Te doping, respectively. In each case the Dirac point is located less than $0.16 \mathrm{eV}$ above the Fermi level, reflecting weakly p-doped silicene. The position of the Dirac point follows the same trend as the band gap, which suggests that the value of the band gap is determined by the amount of charge redistribution between silicene and substrate. Indeed, the internal electric field created at the interface breaks the symmetry of the two silicene sublattices, which opens the band gap.

The charge density difference $\Delta \rho=\rho_{\text {hyb }}+\rho_{\text {sil }}+\rho_{\text {sub }}\left(\rho_{\text {hyb }}, \rho_{\text {sil }}\right.$ and $\rho_{\text {sub }}$ being the charge densities of the hybrid system, silicene and substrate, respectively) due to interaction of silicene with $\mathrm{WSe}_{2}$ is shown in Fig. 6 for the cases without and with a lateral $1 / 3$ shift. Both $\rho_{\text {sil }}$ and $\rho_{\text {sub }}$ are calculated with the same parameters as $\rho_{\text {hyb }}$. From left to right Fig. 6 shows an enhanced charge density redistribution at the interface of the two subsystems for the unshifted structure (upper row), reflecting the growing interaction (the number of valence electrons grows along the series $\mathrm{S}-\mathrm{Se}-\mathrm{Te}$ ). Charge also shifts within the silicene sheet from the lower sublattice to the upper sublattice. Under the lateral shift (lower row) the charge density redistribution at the interface is substantially weaker.

The structure of high-buckled silicene on $\mathrm{WSe}_{2}$ is shown in Fig. 7 for different locations of the Si atoms, namely on top of $\mathrm{W}$, Se and the hollow site. The first case turns out to be energetically favorable by 10 and $3 \mathrm{meV}$ per atom with respect (a)

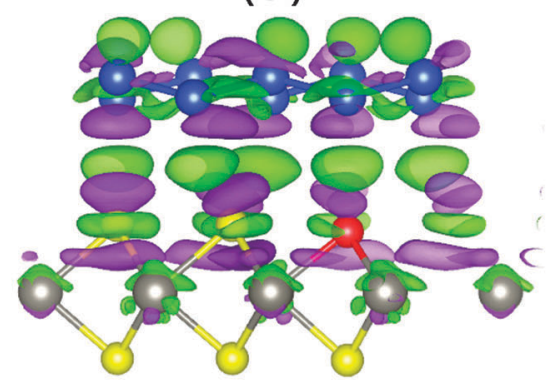

(d)

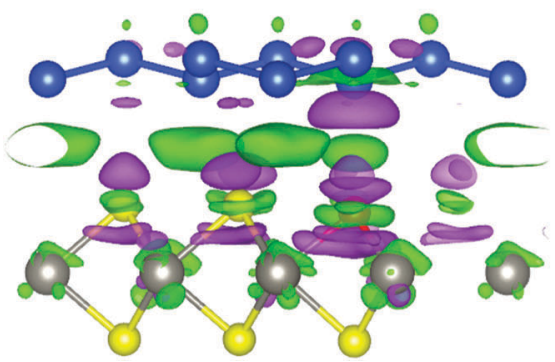

(b)

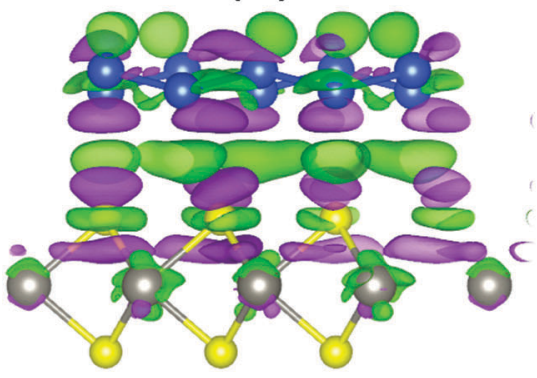

(e)

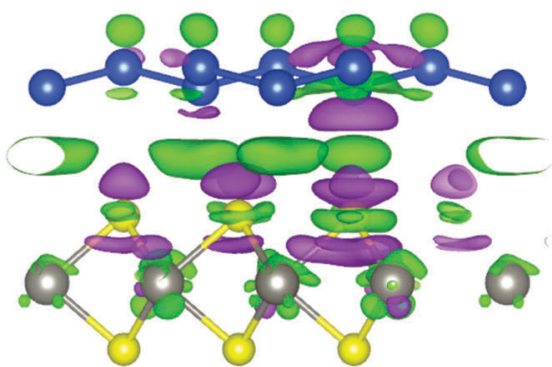

(c)

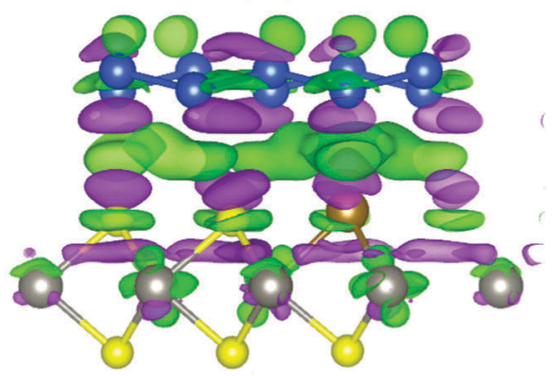

(f)

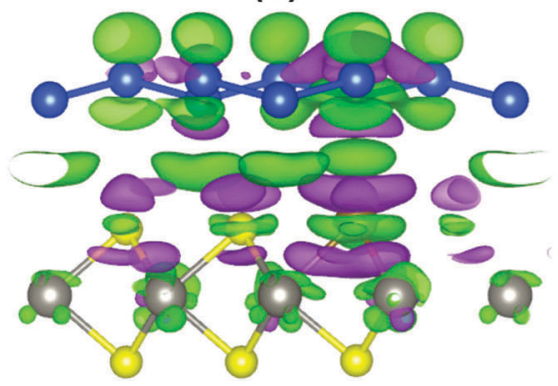

Fig. 6 Charge density difference due to the interaction of low-buckled silicene with (a, d) S doped, (b, e) pristine and (c, f) Te doped WSe 2 without lateral shift (upper row) and with a $1 / 3$ shift (lower row). Green (purple) color represents charge accumulation (depletion), where the isosurfaces refer to isovalues of $3 \times 10^{-4}$ electrons per bohr ${ }^{3}$. The W, Se, Si, S and Te atoms are shown in gray, yellow, blue, red and brown color. 
(a)

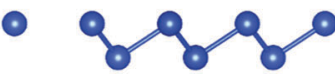

- 989

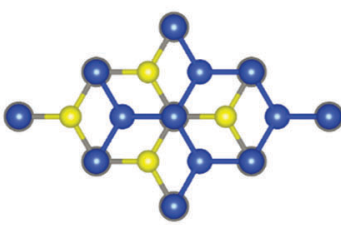

(b)

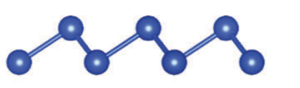

西

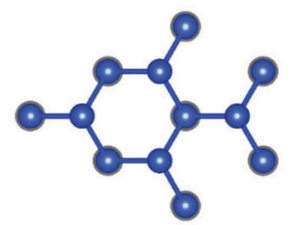

(c)

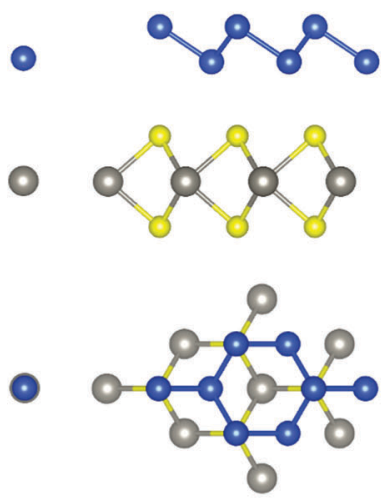

(d)

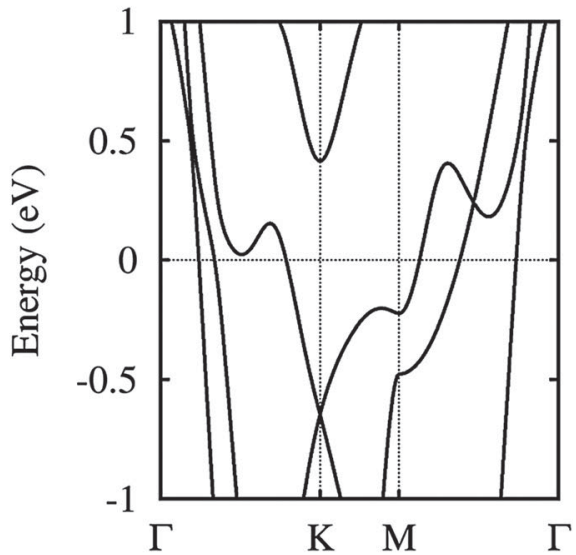

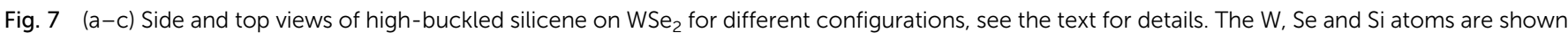
in gray, yellow and blue color. (d) Band structure of the lowest energy configuration (a).

to the other cases, respectively. The Si buckling height is found to be $1.3 \AA$, which is smaller than in the case of high-buckled silicene on the $\mathrm{MoS}_{2}$ substrate $(2.0 \AA)$ due to a reduced lattice compression. ${ }^{37}$ The larger binding energy (195 meV) as compared to low-buckled silicene $(122 \mathrm{meV})$ is a consequence of the smaller distance to the substrate (2.55 ̊instead of $3.20 \AA$ ). This strong interaction destroys the Dirac cone, see Fig. $7(d)$, in contrast to the low-buckled system and similar to previous results on the $\mathrm{MoS}_{2}$ substrate. ${ }^{37,42}$

\section{Conclusions}

The stability and electronic properties of silicene on the semiconducting $\mathrm{WSe}_{2}$ substrate have been investigated using firstprinciples calculations, motivated by the minimal lattice mismatch. The undoped substrate suffers from the structural uncertainty coming from the possibility of lateral shifts between the component systems due to small translation energy barriers. Therefore, electronic structures ranging from metallic to semiconducting silicene are encountered and can fluctuate even at low temperature. On the other hand, silicene on $\mathrm{S}$ and Te doped $\mathrm{WSe}_{2}$ turns out to be stable metallic and semiconducting, respectively, because of different interaction strengths. The translation energy barrier and binding energy for Te doping amount to $7 \mathrm{meV}$ and $135 \mathrm{meV}$, respectively. The Dirac physics of silicene is preserved with the Dirac point located $160 \mathrm{meV}$ above the Fermi level due to charge redistribution. A band gap of $340 \mathrm{meV}$ is achieved, which withstands thermal fluctuations at room and elevated temperatures and results in an improved applicability of the proposed hybrid system.

Using $\mathrm{WSe}_{2}$ as a support will open access to the unique properties of silicene, which so far could not be utilized as they were always perturbed by the substrate. Of particular interest are the unusual performances of silicene-based spintronic devices ${ }^{8}$ and the potential to obtain ultra high speed ( $\mathrm{THz}$ frequency range) field-effect transistors. Such field-effect transistors show a high on/off current ratio ${ }^{43}$ but require a complicated preparation procedure. ${ }^{44}$ All this is only possible if the fundamental shortcomings of the current substrates are resolved. On the one hand, the Dirac cone has to be preserved, which excludes many potential candidates. On the other hand, the lattice mismatch has to be small to avoid strain effects on silicene, the termination must not cause complications, and the band gap must be intrinsically large enough to operate at standard temperatures. All these criteria can be achieved by utilizing $\mathrm{WSe}_{2}$ to exploit the intrinsic properties of silicene, thus avoiding, for example, the large supply voltage required when adatoms are used to open the band gap. ${ }^{45}$ It should be noted that silicene already has been grown epitaxially on $\mathrm{MoS}_{2},{ }^{37}$ which is isostructural to $\mathrm{WSe}_{2}$ and has very similar physical and chemical properties, so that the same preparation method can be expected to be successful.

\section{Acknowledgements}

Research reported in this publication was supported by the King Abdullah University of Science and Technology (KAUST).

\section{References}

1 K. Takeda and K. Shiraishi, Theoretical Possibility of Stage Corrugation in Si and Ge Analogs of Graphite, Phys. Rev. B: Condens. Matter Mater. Phys., 1994, 50, 14916-14922.

2 E. Durgun, S. Tongay and S. Ciraci, Silicon and III-V Compound Nanotubes: Structural and Electronic Properties, Phys. Rev. B: Condens. Matter Mater. Phys., 2005, 72, 075420.

3 N. J. Roome and J. D. Carey, Beyond Graphene: Stable Elemental Monolayers of Silicene and Germanene, ACS Appl. Mater. Interfaces, 2014, 6, 7743-7750.

4 S. Cahangirov, M. Topsakal, E. Aktürk, H. Şahin and S. Ciraci, Two- and One-Dimensional Honeycomb Structures of Silicon and Germanium, Phys. Rev. Lett., 2009, 102, 236804.

5 K. S. Novoselov, A. K. Geim, S. V. Morozov, D. Jiang, Y. Zhang, S. V. Dubonos, I. V. Grigorieva and A. A. Firsov, 
Electric Field Effect in Atomically Thin Carbon Films, Science, 2004, 306, 666-669.

6 A. H. Castro Neto, F. Guinea, N. M. R. Peres, K. S. Novoselov and A. K. Geim, The Electronic Properties of Graphene, Rev. Mod. Phys., 2009, 81, 109-162.

7 K. S. Novoselov, V. I. Fal'ko, L. Colombo, P. R. Gellert, M. G. Schwab and K. Kim, A Roadmap for Graphene, Nature, 2012, 490, 192-200.

8 C. Liu, W. Feng and Y. Yao, Quantum Spin Hall Effect in Silicene and Two-Dimensional Germanium, Phys. Rev. Lett., 2011, 107, 076802.

9 A. Fleurence, R. Friedlein, T. Ozaki, H. Kawai, Y. Wang and Y. Yamada-Takamura, Experimental Evidence for Epitaxial Silicene on Diboride Thin Films, Phys. Rev. Lett., 2012, 108, 245501.

10 L. Meng, Y. Wang, L. Zhang, S. Du, R. Wu, L. Li, Y. Zhang, G. Li, H. Zhou, W. A. Hofer and H. Gao, Buckled Silicene Formation on $\operatorname{Ir}(111)$, Nano Lett., 2013, 13, 685-690.

11 P. Vogt, P. De Padova, C. Quaresima, J. Avila, E. Frantzeskakis, M. C. Asensio, A. Resta, B. Ealet and G. Le Lay, Silicene: Compelling Experimental Evidence for Graphene like TwoDimensional Silicon, Phys. Rev. Lett., 2012, 108, 155501.

12 D. Tsoutsou, E. Xenogiannopoulou, E. Golias, P. Tsipas and A. Dimoulas, Evidence for Hybrid Surface Metallic Band in (4 × 4) Silicene on Ag(111), Appl. Phys. Lett., 2013, 103, 231604.

13 D. Kaltsas, L. Tsetseris and A. Dimoulas, Structural Evolution of Single-Layer Films during Deposition of Silicon on Silver: A First-Principles Study, J. Phys.: Condens. Matter, 2012, 24, 442001.

14 C.-L. Lin, R. Arafune, K. Kawahara, M. Kanno, N. Tsukahara, E. Minamitani, Y. Kim, M. Kawai and N. Takagi, SubstrateInduced Symmetry Breaking in Silicene, Phys. Rev. Lett., 2013, 110, 076801.

15 Y. Wang and H. Cheng, Absence of a Dirac Cone in Silicene on Ag(111): First-Principles Density Functional Calculations with a Modified Effective Band Structure Technique, Phys. Rev. B: Condens. Matter Mater. Phys., 2013, 87, 245430.

16 Y. Ding and Y. Wang, Electronic Structures of Silicene/GaS Heterosheets, Appl. Phys. Lett., 2013, 103, 043114.

17 S. Kokott, L. Matthes and F. Bechstedt, Silicene on Hydrogen-Passivated $\mathrm{Si}(111)$ and $\mathrm{Ge}(111)$ Substrates, Phys. Status Solidi RRL, 2013, 7, 538-541.

18 S. Kokott, P. Pugradt, L. Matthes and F. Bechstedt, Nonmetallic Substrates for Growth of Silicene: An $A b$ Initio Prediction, J. Phys.: Condens. Matter, 2014, 26, 185002.

19 N. Gao, J. Li and Q. Jiang, Bandgap Opening in Silicene: Effect of Substrates, Chem. Phys. Lett., 2014, 592, 222-226.

20 H. Liu, J. Gao and J. Zhao, Silicene on Substrates: A Way to Preserve or Tune Its Electronic Properties, J. Phys. Chem. C, 2013, 117, 10353-10359.

$21 \mathrm{~J}$. Zhu and U. Schwingenschlögl, Structural and Electronic Properties of Silicene on $\mathrm{MgX}_{2}(\mathrm{X}=\mathrm{Cl}, \mathrm{Br}$, and I), ACS Appl. Mater. Interfaces, 2014, 6, 11675-11681.

22 L. Huang, Q. Yue, J. Kang, Y. Li and J. Li, Tunable Band Gaps in Graphene/GaN van der Waals Heterostructures, J. Phys.: Condens. Matter, 2014, 26, 295304.
23 K. Tang, R. Qin, J. Zhou, H. Qu, J. Zheng, R. Fei, H. Li, Q. Zheng, Z. Gao and J. Lu, Electric-Field-Induced Energy Gap in Few-Layer Graphene, J. Phys. Chem. C, 2011, 115, 9458-9464.

24 X. Zhong, Y. K. Yap, R. Pandey and S. P. Karna, FirstPrinciples Study of Strain-Induced Modulation of Energy Gaps of Graphene/BN and BN Bilayers, Phys. Rev. B: Condens. Matter Mater. Phys., 2011, 83, 193403.

25 J. Sławińska, I. Zasada and Z. Klusek, Energy Gap Tuning in Graphene on Hexagonal Boron Nitride Bilayer System, Phys. Rev. B: Condens. Matter Mater. Phys., 2010, 81, 155433.

26 Z. Ni, Q. Liu, K. Tang, J. Zheng, J. Zhou, R. Qin, Z. Gao, D. Yu and J. Lu, Tunable Bandgap in Silicene and Germanene, Nano Lett., 2012, 12, 113-118.

27 M. Ye, R. Quhe, J. Zheng, Z. Ni, Y. Wang, Y. Yuan, G. Tse, J. Shi, Z. Gao and J. Lu, Tunable Band Gap in Germanene by Surface Adsorption, Physica E, 2014, 59, 60-65.

28 Y. Liu, H. Shu, P. Liang, D. Cao, X. Chen and W. Lu, Structural, Electronic, and Optical Properties of Hydrogenated Few-Layer Silicene: Size and Stacking Effects, J. Appl. Phys., 2013, 114, 094308.

29 R. Quhe, R. Fei, Q. Liu, J. Zheng, H. Li, C. Xu, Z. Ni, Y. Wang, D. Yu, Z. Gao and J. Lu, Tunable and Sizable Band Gap in Silicene by Surface Adsorption, Sci. Rep., 2012, 2, 853.

30 Z. Ni, H. Zhong, X. Jiang, R. Quhe, G. Luo, Y. Wang, M. Ye, J. Yang, J. Shi and J. Lu, Tunable Band Gap and Doping Type in Silicene by Surface Sdsorption: Towards Tunneling Transistors, Nanoscale, 2014, 6, 7609-7618.

31 M. Traving, M. Boehme, L. Kipp, M. Skibowski, F. Starrost, E. E. Krasovskii, A. Perlov and W. Schattke, Electronic Structure of $\mathrm{WSe}_{2}$ : A Combined Photoemission and Inverse Photoemission Study, Phys. Rev. B: Condens. Matter Mater. Phys., 1997, 55, 10392-10399.

32 E. Scalise, M. Houssa, E. Cinquanta, C. Grazianetti, B. van den Broek, G. Pourtois, A. Stesmans, M. Fanciulli and A. Molle, Engineering the Electronic Properties of Silicene by Tuning the Composition of $\mathrm{MoX}_{2}$ and $\operatorname{GaX}(\mathrm{X}=\mathrm{S}$, Se, Te) Chalchogenide Templates, 2D Mater., 2014, 1, 011010.

33 G. Kresse and D. Joubert, From Ultrasoft Pseudopotentials to the Projector Augmented-Wave Method, Phys. Rev. B: Condens. Matter Mater. Phys., 1999, 59, 1758-1775.

34 J. P. Perdew, K. Burke and M. Ernzerhof, Generalized Gradient Approximation Made Simple, Phys. Rev. Lett., 1996, 77, 3865-3868.

35 S. Grimme, Semiempirical GGA-Type Density Functional Constructed with a Long-Range Dispersion Correction, J. Comput. Chem., 2006, 27, 1787-1799.

36 A. Kumar and P. Ahluwalia, Electronic Structure of Transition Metal Dichalcogenides Monolayers $1 \mathrm{H}-\mathrm{MX}_{2}(\mathrm{M}=\mathrm{Mo}$, $\mathrm{W} ; \mathrm{X}=\mathrm{S}$, Se, Te) from Ab-Initio Theory: New Direct Band Gap Semiconductors, Eur. Phys. J. B, 2012, 85, 186.

37 D. Chiappe, E. Scalise, E. Cinquanta, C. Grazianetti, B. van den Broek, M. Fanciulli, M. Houssa and A. Molle, TwoDimensional Si Nanosheets with Local Hexagonal Structure on a $\mathrm{MoS}_{2}$ Surface, Adv. Mater., 2014, 26, 2096-2101.

38 W. Zhao, Z. Ghorannevis, K. K. Amara, J. R. Pang, M. Toh, X. Zhang, C. Kloc, P. H. Tan and G. Eda, Lattice Dynamics in 
Mono- and Few-Layer Sheets of $\mathrm{WS}_{2}$ and $\mathrm{WSe}_{2}$, Nanoscale, 2013, 5, 9677-9683.

39 N. Gao, J. C. Li and Q. Jiang, Tunable Band Gaps in Silicene-MoS Heterobilayers, Phys. Chem. Chem. Phys., 2014, 16, 11673-11678.

40 H. Liu, N. Han and J. Zhao, Band Gap Opening in Bilayer Silicene by Alkali Metal Intercalation, J. Phys.: Condens. Matter, 2014, 26, 475303.

41 P. Zhao, D. Kiriya, A. Azcatl, C. Zhang, M. Tosun, Y.-S. Liu, M. Hettick, J. S. Kang, S. McDonnell, S. KC, J. Guo, K. Cho, R. M. Wallace and A. Javey, Air Stable p-Doping of $\mathrm{WSe}_{2}$ by Covalent Functionalization, ACS Nano, 2014, 8, 10808-10814.

$42 \mathrm{~L}$. Li and M. Zhao, Structures, Energetics, and Electronic Properties of Multifarious Stacking Patterns for High-Buckled and Low-Buckled Silicene on the $\mathrm{MoS}_{2}$ Substrate, J. Phys. Chem. C, 2014, 118, 19129-19138.

43 Y. F. Zhu, Q. Q. Dai, M. Zhao and Q. Jiang, Physicochemical Insight into Gap Openings in Graphene, Sci. Rep., 2013, 3, 1524.

44 L. Tao, E. Cinquanta, D. Chiappe, C. Grazianetti, M. Fanciulli, M. Dubey, A. Molle and D. Akinwande, Silicene Field-Effect Transistors Operating at Room Temperature, Nat. Nanotechnol., 2015, 10, 227-231.

45 F. Xia, D. B. Farmer, Y.-M. Lin and P. Avouris, Graphene Field-Effect Transistors with High On/Off Current Ratio and Large Transport Band Gap at Room Temperature, Nano Lett., 2010, 10, 715-718. 